PROCEEDINGS OF THE

AMERICAN MATHEMATICAL SOCIETY

Volume 129, Number 8, Pages 2409-2417

S 0002-9939(01)05910-X

Article electronically published on January 17, 2001

\title{
ISOMETRIC SUBMERSIONS OF FINSLER MANIFOLDS
}

\author{
J. C. ÁlVAREZ PAIVA AND C. E. DURÁN \\ (Communicated by Christopher Croke) \\ Dedicated to Enrique Planchart
}

\begin{abstract}
The notion of isometric submersion is extended to Finsler spaces and it is used to construct examples of Finsler metrics on complex and quaternionic projective spaces all of whose geodesics are (geometrical) circles.
\end{abstract}

\section{INTRODUCTION}

In this paper we extend the theory of Riemannian submersions to Finsler spaces. It may be safely said that the aim of the theory is to construct interesting examples of Finsler manifolds or, if the reader prefers a different terminology, of convex Hamiltonian systems. Our original goal was to construct Finsler metrics on complex projective space whose geodesics coincide with those of the Fubini-Study metric. In this endeavour we failed, but nevertheless we were able to find a large class of Finsler metrics on complex and quaternionic projective spaces all of whose geodesics are (geometrical) circles. Besides the construction of these metrics, we also study the relation between Finsler submersions and symplectic reduction and show that, like their Riemannian counterparts, Finsler submersions are curvature non-decreasing.

\section{Isometric Submersions of Minkowski spaCeS}

The infinitesimal version of Riemannian submersions, isometric submersions of Euclidean spaces, is too trivial to deserve mention. Nevertheless, understanding the simple geometry of isometric submersions of normed spaces is the key to extending the theory to Finsler manifolds. In this section we study isometric submersions of normed spaces from the point of view of the spaces themselves and from that of their duals. The latter point of view will allow us in section 5 to relate isometric submersions with symplectic reduction. The proofs of the propositions in this section are all elementary and are left to the interested reader.

Definition 2.1. A surjective linear map $\pi: V_{1} \rightarrow V_{2}$ between two normed spaces $\left(V_{1},\|\cdot\|_{1}\right)$ and $\left(V_{2},\|\cdot\|_{2}\right)$ is said to be an isometric submersion if the image of the closed unit ball on $V_{1}$ under the map $\pi$ equals the closed unit ball on $V_{2}$.

Received by the editors December 14, 1999.

1991 Mathematics Subject Classification. Primary 53C22, 53C60; Secondary 53D20.

Key words and phrases. Finsler manifolds, isometric submersions, symplectic reduction.

The first author was partially supported by a Crédit aux Chercheurs from the FNRS.

(C)2001 American Mathematical Society 
Usually, the normed space $\left(V_{1},\|\cdot\|_{1}\right)$ and the surjective linear map $\pi: V_{1} \rightarrow V_{2}$ are given, while the norm on $V_{2}$ is defined by projecting the closed unit ball in $V_{1}$. The next proposition gives the analytical description of the subduced norm.

Proposition 2.1. If $\pi: V_{1} \rightarrow V_{2}$ is an isometric submersion between the normed spaces $\left(V_{1},\|\cdot\|_{1}\right)$ and $\left(V_{2},\|\cdot\|_{2}\right)$, then for every vector $w \in V_{2}$

$$
\|w\|_{2}=\inf \left\{\|v\|_{1}: \pi(v)=w\right\} .
$$

In other words, the norm of $w$ equals the distance from the origin to the affine subspace $\pi^{-1}(w) \subset V_{1}$.

It is clear that if $v$ is any vector in $V_{1}$, then $\|\pi(v)\|_{2} \leq\|v\|_{1}$. The vectors for which the equality holds are said to be horizontal and form the horizontal cone in $V_{1}$. Notice that if the norm on $V_{1}$ is Euclidean, then the horizontal cone is the subspace orthogonal to the kernel of $\pi$. The fact that, in general, the horizontal cone is not a subspace constitutes one of the main differences between isometric submersions of Riemannian and Finsler manifolds.

In Finsler geometry, one is interested in a very special class of normed spaces. In extrinsic terms, these are spaces where the norm is smooth and the unit sphere has positive principal curvatures for some (and therefore any) auxiliary Euclidean structure. The intrinsic definition is as follows:

Let $V$ be a vector space and let $\varphi: V \rightarrow[0, \infty)$ be a norm which is smooth outside the origin. At each point $v \in V \backslash 0$ define the bilinear form $g_{\varphi}(v)$ as follows: let $w_{1}$ and $w_{2}$ be two vectors in $V$ and let $\alpha(s, t)$ be a smooth vector-valued function such that $\alpha(0,0)=v, \frac{\partial \alpha}{\partial s}(0,0)=w_{1}$, and $\frac{\partial \alpha}{\partial t}(0,0)=w_{2}$. Set

$$
g_{\varphi}(v)\left(w_{1}, w_{2}\right):=\frac{1}{2} \frac{\partial^{2}}{\partial s \partial t} \varphi^{2}(\alpha(s, t))_{\mid(0,0)} .
$$

Definition 2.2. A smooth norm $\varphi: V \rightarrow[0, \infty)$ is said to be a Minkowski norm if for every nonzero vector $v$ the bilinear form $g_{\varphi}(v)$ is positive definite. A vector space provided with a Minkowski norm will be called a Minkowski space.

When the vector $v$ belongs to the unit sphere $S(V)$, we will refer to $g_{\varphi}(v)$ as the osculating Euclidean structure at $v$ and to the ellipsoid

$$
E_{v}=\left\{w \in V: g_{\varphi}(v)(w, w) \leq 1\right\}
$$

as the osculating ellipsoid of $S(V)$ at $v$.

Proposition 2.2. Let $\pi:\left(V_{1}, \varphi_{1}\right) \rightarrow\left(V_{2}, \varphi_{2}\right)$ be a submersion of Minkowski spaces. If $v \in V_{1}$ is a horizontal vector of norm one, then the osculating ellipsoid of $S\left(V_{1}\right)$ at $v$ projects onto the osculating ellipsoid of $S\left(V_{2}\right)$ at $\pi(v)$. In other words, the map $\pi$ is an isometric submersion between the Euclidean spaces $\left(V_{1}, g_{\varphi_{1}}(v)\right)$ and $\left(V_{2}, g_{\varphi_{2}}(\pi(v))\right)$.

Note that by homogeneity, if $v \in V_{1}$ is a horizontal vector (not necessarely of norm one), then $\pi$ is still an isometric submersion between the Euclidean spaces $\left(V_{1}, g_{\varphi_{1}}(v)\right)$ and $\left(V_{2}, g_{\varphi_{2}}(\pi(v))\right)$.

In Minkowski spaces, the horizontal cone can be descibed in various ways. For example, it is easy to see that the intersection of the horizontal cone and the unit sphere $S\left(V_{1}\right)$ is the singular set of the map $\pi$ restricted to $S\left(V_{1}\right)$. Note also that if $v$ is a nonzero horizontal vector, then the tangent space of the cone at $v$ is the subspace orthogonal to the kernel of $\pi$ with respect to the inner product $g_{\varphi_{1}}(v)$. 
Isometric submersions and duality. Recall that if $(V,\|\cdot\|)$ is a normed space, then the dual vector space $V^{*}$ inherits a natural norm defined by the equation

$$
\|\xi\|^{*}:=\sup \{|\xi \cdot v|:\|v\| \leq 1\} .
$$

The next result shows that submersions are dual to isometric embeddings of normed spaces.

Proposition 2.3. If $\pi: V_{1} \rightarrow V_{2}$ is an isometric submersion, then the dual map $\pi^{*}: V_{2}^{*} \rightarrow V_{1}^{*}$, defined by

$$
\pi^{*}(\eta) \cdot v=\eta \cdot \pi(v)
$$

whenever $\eta \in V_{2}^{*}$ and $v \in V_{1}$, is an isometric embedding.

If $(V, \varphi)$ is a Minkowski space, the map $v \mapsto g_{\varphi}(v)(v, \cdot)$ is a diffeomorphism between $V \backslash 0$ and $V^{*} \backslash 0$ that is homogeneous of degree one. In what follows, the continuous extension of this map to all of $V$ will be called the Legendre transform and will be denoted by $\mathcal{L}$. Geometrically, the Legendre transform can be defined as follows: if $v$ is a unit vector, then $\mathcal{L}(v)$ is the unique covector $\xi$ such that

$$
\{w \in V: \xi \cdot v=1\}=T_{v} S(V) .
$$

Using the Legendre transform, we can see that the horizontal cone is really a linear subspace in disguise:

Proposition 2.4. Let $\pi: V_{1} \rightarrow V_{2}$ be an isometric submersion of Minkowski spaces. If $\pi^{*}: V_{2}^{*} \rightarrow V_{1}^{*}$ is the dual map, then the subspace $\pi^{*}\left(V_{2}^{*}\right) \subset V_{1}^{*}$ is the annihilator of $\operatorname{Ker}(\pi) \subset V_{1}$. Moreover, the image of this subspace under the Legendre transform $\mathcal{L}: V_{1}^{*} \rightarrow V_{1}$ is the horizontal cone.

\section{ISOMETRIC SUBMERSIONS OF FinsleR MANIFOLDS}

In this section we extend the notion of isometric submersion to Finsler manifolds. We start with a few basic definitions.

Definition 3.1. Let $M$ be a smooth manifold and let $T M \backslash 0$ denote its tangent bundle with the zero section deleted. A Finsler metric on $M$ is a smooth positive function

$$
\varphi: T M \backslash 0 \longrightarrow \mathbb{R}
$$

such that for each point $m \in M$ the restriction of $\varphi$ to $T_{m} M$ is a Minkowski norm.

Definition 3.2. A submersion $\rho: M \rightarrow N$ between Finsler manifolds is said to be isometric if for every point $m \in M$ the differential

$$
D_{m} \rho: T_{m} M \longrightarrow T_{\rho(m)} N
$$

is an isometric submersion of Minkowski spaces.

Examples. 1. A large class of examples of isometric submersions can be constructed by taking a principal fiber bundle $\rho: P \rightarrow M$ with structure group $G$, together with a $G$-invariant Finsler metric $\phi$ on $P$. By the invariance of $\phi$, the image under the differential of $\rho$ of the unit ball in any tangent space of $P$ depends only on the fiber. It is possible then to define a Finsler metric $\hat{\phi}$, the subduced metric, on $M$ such that the map $\rho$ is an isometric submersion. Explicit examples of this construction will be considered in the next section. 
2. Let $G$ be a Lie group, let $K \subset G$ be a subgroup, and let $K \backslash G$ denote the set of right cosets $K g, g \in G$. The natural projection $\rho: G \rightarrow K \backslash G$ defines a principal fiber bundle with structure group $K$ acting on the left. A left-invariant Finsler metric $\varphi$ on $G$ is also left-invariant for the action of $K$ on $G$ and, therefore, induces a Finsler metric on $K \backslash G$ that is not necessarily invariant under the right action of $G$. This particular case of example 1 will be treated in section 5 from the Hamiltonian point of view.

While there are in general no horizontal subspaces in Finsler submersions, we can talk of horizontal vectors, horizontal curves, and horizontal lifts of curves and vector fields.

Definition 3.3. Let $\rho: M \rightarrow N$ be an isometric submersion. An immersed curve $\gamma:[a, b] \rightarrow M$ is said to be horizontal if for every $t \in(a, b)$ the velocity vector $\dot{\gamma}(t)$ belongs to the horizontal cone in $T_{\gamma(t)} M$. A curve $\gamma:[a, b] \rightarrow M$ is said to be a horizontal lift of an immersed curve $\sigma:[a, b] \rightarrow N$ if $\gamma$ is horizontal and $\rho \circ \gamma=\sigma$.

Note that if $\sigma:[a, b] \rightarrow N$ is an immersed curve and $m$ is a point in $\rho^{-1}(\sigma(a))$, then there exists a unique horizontal lift of $\sigma$ passing through $m$. Moreover, it follows immediately from the definitions that if $\gamma$ is a smooth curve on $M$, then the length of $\gamma$ is greater than or equal to the length of $\rho \circ \gamma$, with equality holding if and only if $\gamma$ is horizontal. These trivial remarks lead up to the following important fact:

Theorem 3.1. An immersed curve on $N$ is a geodesic if and only if any of its horizontal lifts is a geodesic on $M$. In particular, the geodesics of $N$ are precisely the projections of horizontal geodesics on $M$.

Proof. Let us start by proving that if $\sigma:[a, b] \rightarrow N$ is a geodesic, then any of its horizontal lifts is also geodesic. Since being a geodesic is a local property, it is sufficient to consider the case where $\sigma$ is the unique minimal geodesic joining its endpoints.

Let $\gamma:[a, b] \rightarrow M$ be a horizontal lift of $\sigma$. It is not hard to see that $\gamma$ is the shortest curve on $M$ joining its endpoints. Indeed, if $\gamma^{\prime}$ were a shorter curve joining $\gamma(a)$ to $\gamma(b)$, then the projection of $\gamma^{\prime}$ to $N$ would be a curve joining $\sigma(a)$ to $\sigma(b)$ that is shorter than $\sigma$. Since this is impossible, $\gamma$ is a geodesic.

Let us now prove that if a horizontal lift of an immersed curve in $N$ is a geodesic, then the curve is itself a geodesic. Indeed, we have just seen that for every point $m \in M$ and horizontal vector $v \in T_{m} M$ we can construct a horizontal geodesic passing through $m$ with velocity $v$ as the horizontal lift of a geodesic on $N$. Since geodesics are uniquely determined by their initial condition, this implies that a geodesic on $M$ that starts horizontally is in fact the horizontal lift of a geodesic on $N$.

\section{Finsler metrics ON $\mathbb{C} P^{n}$ AND $\mathbb{H} P^{n}$ ALL OF WHOSE GEODESICS ARE CIRCLES}

The Finsler metrics in this section are constructed by making use of the Hopf fibrations and a class of metrics on the sphere all of whose geodesics are great circles. A strikingly simple and elegant construction of this class of metrics has been given by Busemann (see [7, 9, 11], or [6, 5] for an approach closer to the one in this paper): 
Theorem 4.1 (The Busemann construction). If $\Phi$ be a smooth positive measure on the space $S^{n *}$ of totally geodesic hyperspheres in the $n$-sphere, then there exists a Finsler metric $\phi$ on $S^{n}$ such that for every smooth curve $\gamma:[a, b] \rightarrow S^{n}$

$$
\int_{\xi \in S^{n *}} \#(\xi \cap \gamma) \Phi=\int_{\gamma} \phi,
$$

where $\#(\xi \cap \gamma)$ denotes the number of intersections of the hypersphere $\xi$ with the curve $\gamma$. Moreover, as a consequence of this equation, the geodesics of $\left(S^{n}, \phi\right)$ are great circles.

Note that by choosing the measure $\Phi$ to be invariant under the action of a certain subgroup of $S O(n+1)$, we obtain Finsler metrics with the same invariance. In particular, we can easily construct Finsler metrics on $S^{2 n+1}$ (resp. $S^{4 n+3}$ ) whose geodesics are great circles and which are invariant under the Hopf action of $S^{1}$ (resp. $\left.S^{3}\right)$.

Let $\rho: S^{2 n+1} \rightarrow \mathbb{C} P^{n}$ be the Hopf fibration considered as a principal bundle with structure group $S^{1}$. If $\phi$ is an $S^{1}$-invariant Finsler metric on $S^{2 n+1}$, we may construct the subduced metric on $\mathbb{C} P^{n}$. Obviously, the same can be done on $\mathbb{H} P^{n}$ if we consider the Hopf fibration $\rho: S^{4 n+3} \rightarrow \mathbb{H} P^{n}$ and $S^{3}$-invariant metrics on $S^{4 n+3}$.

Theorem 4.2. Let $\phi$ be a Finsler metric on $S^{2 n+1}$ (resp. $S^{4 n+3}$ ) such that its geodesics are great circles. If $\phi$ is invariant under the action of $S^{1}$ (resp. $S^{3}$ ), then the subduced metric on $\mathbb{C} P^{n}$ (resp. $\left.\mathbb{H} P^{n}\right)$ is such that all of its geodesics are circles.

By Theorem 3.1, the geodesics on $\mathbb{C} P^{n}$ (resp. $\mathbb{H} P^{n}$ ) are the images of great circles under the Hopf fibration. The proof of the preceding theorem is then a consequence of the following well-known result:

Lemma 4.3. The image of a great circle on $S^{2 n+1}$ (resp. $S^{4 n+3}$ ) under the Hopf fibration $\rho: S^{2 n+1} \rightarrow \mathbb{C} P^{n}$ (resp. $\left.\rho: S^{4 n+1} \rightarrow \mathbb{H} P^{n}\right)$ is a circle.

Proof. We shall prove the lemma for the Hopf fibration $\rho: S^{2 n+1} \rightarrow \mathbb{C} P^{n}$. The proof for the quaternionic case is basically identical: it suffices for the reader to replace $S^{2 n+1}$ by $S^{4 n+3}, \mathbb{C}$ by $\mathbb{H}$, and the word complex by the word quaternionic.

We start by noting that any great circle in $S^{2 n+1} \subset \mathbb{C}^{n+1}$ is contained in 2dimensional complex subspace. It is therefore sufficient to prove the lemma in the case of the fibration

$$
S^{1} \rightarrow S^{3} \stackrel{\pi}{\longrightarrow} S^{2}=\mathbb{C} P^{1}
$$

Since the group $U(2)$ acts transitively $S^{3}$ and sends great circles to great circles, we can assume without loss of generality that the given circle $\gamma$ passes through the point $(1,0) \in \mathbb{C}^{2}$, and $\gamma$ can be parametrized by

$$
\gamma(t)=(\cos (t)+a \sin (t), b \sin (t)),
$$

where $\operatorname{Re}(a)=0$, and $|a|^{2}+|b|^{2}=1$.

If $w: S^{3} \rightarrow \mathbb{C}$ is the map $w\left(z_{1}, z_{2}\right):=z_{2}^{-1} z_{1}$, then the projection $\ell(t)=w \circ \gamma$ produces the straight line

$$
\ell(t)=\frac{\bar{b}}{|b|^{2}} \cot (t)+\frac{\bar{b} a}{|b|^{2}}
$$


Since $w=S t \circ \pi$, where $S t: S^{2} \rightarrow \mathbb{C}$ is the stereographic projection and since the stereographic projection maps circles to generalized circles, it follows that the image of $S t^{-1} \circ \ell=\pi \circ \gamma$ is a circle on the sphere.

Problem 1. Construct Finsler metrics on the Cayley plane all of whose geodesics are circles.

Remark. Theorem 4.2 provides the only known examples of symmetric Finsler metrics on $\mathbb{C} P^{n}$ and $\mathbb{H} P^{n}$ all of whose geodesics are closed. It would be interesting to find other constructions.

\section{ISOMETRIC SUBMERSIONS AND SYMPLECTIC REDUCTION}

In this section we review isometric submersions from the Hamiltonian point of view and uncover their relation with symplectic reduction and momentum maps.

Review of basic notions on symplectic reduction. Very roughly, symplectic reduction is a technique that allows us to construct symplectic manifolds by quotienting out the symmetries of larger ones. We now state the basic definitions and results while referring the reader to pages 402-419 of [2] for the proofs.

Definition 5.1. If $(V, \omega)$ is a symplectic vector space and $W \subset V$ is a subspace, we define

$$
W^{\perp}:=\{v \in V: \omega(v, w)=0 \text { for all vectors } w \in W\} .
$$

The subspace $W$ is said to be isotropic, Lagrangian, or coisotropic depending on whether $W \subset W^{\perp}, W=W^{\perp}$, or $W \supset W^{\perp}$.

The following criterion for a subspace to be coisotropic is often useful in practice.

Proposition 5.1. Let $(V, \omega)$ be a symplectic vector space and let $W \subset V$ be a subspace. If $W$ contains a Lagrangian subspace of $V$, then $W$ is coisotropic.

Proof. If $L \subset W$ is Lagrangian, then $W \supset L=L^{\perp} \supset W^{\perp}$.

Proposition-Definition 5.1. A submanifold $\mathcal{N}$ of a symplectic manifold $(P, \omega)$ is said to be coisotropic if for every $n \in \mathcal{N}, T_{n} \mathcal{N}$ is a coisotropic subspace of $\left(T_{n} P, \omega_{n}\right)$. The distribution $n \mapsto T_{n} \mathcal{N}^{\perp}$, defined on $\mathcal{N}$, is integrable and the induced foliation is called the canonical foliation.

Theorem 5.1. Let $\mathcal{N}$ be a coisotropic submanifold of a symplectic manifold $(P, \omega)$, let $\mathcal{N}_{R}$ denote the space of leaves of the canonical foliation, and let $i: \mathcal{N} \rightarrow P$ and $\pi: \mathcal{N} \rightarrow \mathcal{N}_{R}$ be, respectively, the natural inclusion and the natural projection. If $\mathcal{N}_{R}$ is a smooth manifold, then there exists a unique symplectic form $\omega_{R}$ on $\mathcal{N}_{R}$ defined by the equation $\pi^{*} \omega_{R}=i^{*} \omega$.

Definition 5.2. Let $(P, \omega)$ be a symplectic manifold and let $H: P \rightarrow \mathbb{R}$ be a smooth function. The vector field $X_{H}$ defined by the equation $d H=\omega\left(X_{H}, \cdot\right)$ is called the Hamiltonian vector field of $H$.

Theorem 5.2. Let $\mathcal{N}$ be a coisotropic submanifold of a symplectic manifold $(P, \omega)$ such that the space of leaves of the canonical foliation, $\mathcal{N}_{R}$, is a smooth manifold. If $H: P \rightarrow \mathbb{R}$ is a smooth function that is constant on the leaves of the canonical foliation, then the Hamiltonian vector field $X_{H}$ is tangent to $\mathcal{N}$. Moreover, if $H_{R}$ 
is the induced function on $\mathcal{N}_{R}$, then the Hamiltonian vector fields $X_{H}$ and $X_{H_{R}}$ are related by the equation

$$
X_{H_{R}}=D \pi \circ X_{H},
$$

where $\pi$ is the natural projection from $\mathcal{N}$ to $\mathcal{N}_{R}$.

Note that if the vector field $X_{H}$ defines a flow $F_{t}$, then the above theorem immediately implies that $\mathcal{N}$ is invariant under this flow and that $\pi \circ F_{t}=\hat{F}_{t} \circ \pi$, where $\hat{F}_{t}$ is the flow of $X_{H_{R}}$.

The symplectic geometry of isometric submersions. From the Hamiltonian point of view, a Finsler metric on a manifold $M$ is a smooth function $H: T^{*} M \backslash 0 \rightarrow \mathbb{R}$ such that at every point $m \in M$ the restriction of $H$ to $T_{m}^{*} M$ is a Minkowski norm. Using the standard symplectic form on $T^{*} M$ we may construct the vector field $X_{H}$ whose integral orbits project down to geodesics on $M$.

Proposition 5.2. Let $\rho: M \rightarrow N$ be a submersion of Finsler manifolds and for each $m \in M$ define $\mathcal{N}_{m}$ to be the annihilator of $\operatorname{Ker}\left(D_{m} \rho\right)$. The union, $\mathcal{N}$, of all the $\mathcal{N}_{m}, m \in M$, is a coisotropic submanifold of $T^{*} M$.

Proof. Note that, by definition, the manifold $\mathcal{N}$ is foliated by the co-normals of the fibers of $\rho$. Since co-normals of submanifolds of $M$ are Lagrangian submanifolds of $T^{*} M$, each tangent space of $\mathcal{N}$ contains a Lagrangian subspace and is, therefore, coisotropic.

We shall now see that the space of leaves of the canonical foliation on $\mathcal{N}$ can be naturally identified with the cotangent bundle of $N$. We do this explicitly, by defining a projection $\pi: \mathcal{N} \rightarrow T^{*} N$ which is constant on the leaves and which satisfies the equation $\pi^{*} \omega_{N}=i^{*} \omega_{M}$, where $\omega_{N}$ and $\omega_{M}$ are the canonical symplectic forms on $T^{*} N$ and $T^{*} M$.

If $\xi_{m} \in \mathcal{N}_{m}$, define $\pi\left(\xi_{m}\right) \in T^{*} N$ as the unique covector such that for all $w_{\rho(m)} \in T_{\rho(m)}$

$$
\pi\left(\xi_{m}\right) \cdot w_{\rho(m)}=\xi_{m} \cdot v_{m},
$$

where $v_{m} \in T_{m} M$ is any vector satisfying $D_{m} \rho\left(v_{m}\right)=w_{\rho(m)}$. We note in passing that if $p_{M}$ and $p_{N}$ are the canonical projections, the following diagram commutes:

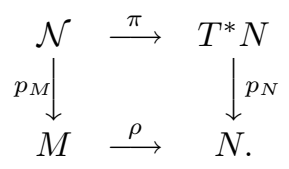

Proposition 5.3. Let $i: \mathcal{N} \rightarrow T^{*} M$ be the natural inclusion and let $\pi: \mathcal{N} \rightarrow T^{*} N$ be the map defined above. If $\alpha_{M}$ and $\alpha_{N}$ are, respectively, the canonical 1-forms on $T^{*} M$ and $T^{*} N$, then $\pi^{*} \alpha_{N}=i^{*} \alpha_{M}$. In particular, $\pi^{*} \omega_{N}=i^{*} \omega_{M}$.

Proof. If $v \in T_{\xi_{m}} \mathcal{N}$,

$$
\pi^{*} \alpha_{N}(v)=\alpha_{N}(D \pi(v))=\pi\left(\xi_{m}\right) \cdot\left(D p_{N}(D \pi(v))\right)=\xi_{m} \cdot u,
$$

where $u$ is any vector such that $D \rho(u)=D p_{N}(D \pi(v))$. By the diagram above, we may choose $u$ to be $D p_{M}(v)$ and therefore

$$
\pi^{*} \alpha_{N}(v)=\xi_{m} \cdot D p_{M}(v)=\alpha_{M}(v) .
$$


As a consequence of this proposition and of the general theory of symplecic reduction, we arrive at the following result:

Theorem 5.3. Let $\rho: M \rightarrow N$ be a submersion of Finsler manifolds. If $M$ is complete and $F_{t}$ denotes the geodesic flow on $T^{*} M$, then $\mathcal{N}$ is invariant under $F_{t}$. Moreover, $N$ is complete and its geodesic flow $\hat{F}_{t}$ satisfies $\hat{F}_{t} \circ \pi=\pi \circ F_{t}$.

This result is, apart from the hypothesis of completeness on $M$, the Hamiltonian version of Theorem 3.1. The construction, given in example 2 of section 3 , of subduced metrics on homogeneous spaces from left-invariant metrics on Lie groups also has a simple Hamiltonian version.

Let $M$ be a homogeneous space under the right action of a Lie group $G$. Consider the lifted Hamiltonian action $T^{*} M \times G \rightarrow T^{*} M$ and its momentum map $J$ : $T^{*} M \longrightarrow \mathcal{G}^{*}$ defined by $J\left(\xi_{m}\right) \cdot X:=\xi_{m} \cdot X_{M}(m)$, where $X$ is any vector in the Lie algebra $\mathcal{G}$ and $X_{M}(m):=d / d t(m \exp (t X))_{\mid t=0}$.

For each $m \in M$, the restriction $J: T_{m}^{*} M \rightarrow \mathcal{G}^{*}$ is a linear embedding and, therefore, if $\varphi^{*}$ is a Minkowski norm on $\mathcal{G}^{*}$, then the collective Hamiltonian $\varphi^{*} \circ J$ defines a Finsler metric on $M$.

Proposition 5.4. Let $(\mathcal{G}, \varphi)$ be the dual of the Minkowski space $\left(\mathcal{G}^{*}, \varphi^{*}\right)$ and let $\varphi$ equally denote the left-invariant Finsler metric on $G$ defined by left translating the norm on the Lie algebra. The Finsler metric defined on $M$ by the Hamiltonian $\varphi^{*} \circ J$ coincides with the subduced metric defined by $\varphi$ and the natural projection $\rho: G \rightarrow M$.

The simple proof is left to the reader.

\section{Submersions And Curvature}

Riemannian submersions are interesting mainly because they allow us to construct examples of Riemannian manifolds while maintaining some control on their geodesics and curvature. In this section we will show that, like their Riemannian counterparts, Finsler submersions are curvature non-decreasing.

We start by defining the Finslerian analogue of the sectional curvature, the so-called flag curvature, which controls the infinitesimal focusing of geodesics via Jacobi's equation. See for example [1] for the deduction of the second variation formula using the flag curvature. The delightfully simple description below is due to Z. Shen and can be found in [10].

If $X$ is a nowhere zero vector field defined on an open subset $\mathcal{O}$ of a Finsler manifold $(M, \varphi)$, then we may associate to $X$ a Riemannian metric on $\mathcal{O}$ by assigning to each point $m \in \mathcal{O}$ the positive definite bilinear form $g_{\varphi}(X(m)): T_{m} M \times T_{m} M \rightarrow \mathbb{R}$ defined as in section 2. A particularly interesting case of this construction is when the integral curves of the vector field are geodesics of the Finsler metric on $M$. These vector fields are called geodesic vector fields.

Proposition-Definition 6.1 (Shen). Let $M$ be a Finsler manifold and let $v_{m} \in$ $T_{m} M$ be a nonzero vector. If $\Pi \subset T_{m} M$ is a two-dimensional subspace containing $v_{m}$ and $X$ is a geodesic vector field defined on a neighbourhood of $m$ such that $X(m)=v_{m}$, then the sectional curvature of the Riemannian metric associated to $X$ at the plane $\Pi$ depends only on the Finsler metric on $M$, the plane $\Pi$, and the vector $v_{m}$. This invariant is called the flag curvature of $M$ at the flag $\left(\Pi, v_{m}\right)$ and is denoted by $K\left(\Pi, v_{m}\right)$. 
If $\rho:(M, \varphi) \rightarrow(N, \hat{\varphi})$ is an isometric submersion of Finsler manifolds and $\hat{X}$ is a nowhere zero geodesic vector field defined on a neighbourhood $\mathcal{O}$ of a point $n \in N$, then the horizontal lift of $\hat{X}$, denoted by $X$, is a geodesic vector field on $\rho^{-1}(\mathcal{O})$ by Theorem 3.1. Moreover, if we provide $\mathcal{O}$ and $\rho^{-1}(\mathcal{O})$ with the Riemannian metrics associated to $\hat{X}$ and $X$, respectively, then we claim that the restriction of $\rho$ to $\rho^{-1}(\mathcal{O})$ is a Riemannian submersion: indeed, for every $m \in M$, since $X_{m}$ is horizontal and $\rho_{*} X_{m}=\hat{X}_{m}$, Proposition 2.2 and its homogeneous extension applies. Then the projection $\rho_{*}$ induces an isometric submersion between the Euclidean spaces $\left(T_{m} M, g_{\varphi}\left(X_{m}\right)\right)$ and $\left(T_{\rho(m)} N, g_{\hat{\varphi}}(X)\right)$, which means that $\rho:\left(\rho^{-1} \mathcal{O}, g_{\varphi}(X)\right) \rightarrow\left(\mathcal{O}, g_{\hat{\varphi}}(X)\right)$ is a Riemannian submersion.

As a consequence of the theory of Riemannian submersions (see, for instance, [8] p. 106), we have that Finsler submersions do not decrease the flag curvature:

Theorem 6.1. Let $\rho: M \rightarrow N$ be a submersion of Finsler manifolds. If $K_{M}$ and $K_{N}$ denote, respectively, the flag curvatures of $M$ and $N$, then

$$
K_{N}\left(\Pi, w_{n}\right) \geq K_{M}\left(\Xi, v_{m}\right),
$$

where $\left(\Xi, v_{m}\right)$ is a horizontal lift of $\left(\Pi, w_{n}\right)$.

\section{REFERENCES}

[1] M. Abate and G. Patrizio, "Finsler Metrics - A Global Approach", Lecture Notes in Mathematics no. 1591, Springer-Verlag, Berlin, 1994. MR 96b:53087

[2] R. Abraham and J. Marsden, "Foundations of Mechanics", Benjamin/Cummings, Reading, Mass. 1978. MR 81e:58025

[3] J.C. Álvarez Paiva, Some problems on Finsler geometry, preprint 1999.

[4] J.C. Álvarez Paiva and C. Durán, "An Introduction to Finsler Geometry", Notas de la Escuela Venezolana de Matématicas, 1998.

[5] J.C. Álvarez Paiva and E. Fernandes, Crofton formulas in projective Finsler spaces, Electronic Research Announcements of the Amer. Math. Soc. 4 (1998), 91-100. MR 99j:53097

[6] J.C. Álvarez Paiva, I.M. Gelfand, and M. Smirnov, Crofton densities, symplectic geometry, and Hilbert's fourth problem, in "Arnold-Gelfand Mathematical Seminars, Geometry and Singularity Theory", V.I. Arnold, I.M. Gelfand, and V.S. Retakh (eds.), Birkhauser, Boston, 1997, pp. 77-92. MR 98a:52005

[7] H. Busemann, Geometries in which planes minimize area, Ann. Mat. Pura Appl. (4) 55 (1961), 171-190. MR 26:715

[8] W. Klingenberg, "Riemannian Geometry", de Gruyter, Berlin, New York, 1982. MR 84j:53001

[9] A.V. Pogorelov, "Hilbert's fourth problem", Scripta Series in Mathematics, Winston and Sons, 1979. MR 80j:53066

[10] Z. Shen, Differential geometry of Spray and Finsler Spaces, preprint, 1999.

[11] Z.I. Szabo, Hilbert's fourth problem I, Adv. in Math. 129 (1986), 185-301. MR 88f:53113

Institut de Mathématique Pure et Appliquée, Université Catholique de Louvain, Chemin du Cyclotron 2, B-1348 Louvain-la-Neuve, Belgium

E-mail address: alvarez@agel.ucl.ac.be

IVIC-Matemáticas, Aptdo. 21287, Caracas 1020A, Venezuela

E-mail address: cduran@cauchy.ivic.ve 